\title{
Inquérito sobre o uso de contenção física em um hospital psiquiátrico de grande porte no Rio de Janeiro
}

\author{
Survey on the use of restraints in a large psychiatric hospital in Rio de Janeiro city
}

Emeli Moura de Araújo1, Eduardo Silveira Martins', Clive Elliot Adams², Evandro Silva Freire Coutinho3o', Gisele Huf'

\section{RESUMO}

Objetivo: Investigar o uso da contenção física em hospital psiquiátrico no Rio de Janeiro. Métodos: Um inquérito foi conduzido em agosto de 2009. As informações - sexo, idade, diagnóstico, ocorrência e duração de contenção física - foram coletadas no prontuário. Resultado: A amostra consistiu em 66 pacientes, dos quais 59\% eram mulheres, com idade média de 44 anos. Durante o período estudado, 24\% dos pacientes foram contidos no leito pelo menos uma vez, mas não é prática corrente o registro detalhado do procedimento. Não ocorreram eventos adversos importantes. Não houve associação entre o uso de contenção e variáveis sociodemográficas e clínicas. Conclusões: A prática de contenção física parece consistente nas emergências psiquiátricas do Rio de Janeiro: esse mesmo percentual foi observado em estudos conduzidos em três hospitais em outros momentos, 2001 e 2004, e situa-se em uma faixa intermediária em relação aos resultados observados em outros países. Não existem estudos randomizados para se fazer uma avaliação objetiva dos benefícios e riscos dessa prática, mas a segurança e a eficácia dessa intervenção deveriam ser objeto do mesmo escrutínio científico normalmente destinado aos outros tratamentos.

\section{Palavras-chave \\ Hospital psiquiátrico, estudo seccional, contenção física, agressão.}

\section{Keywords}

Psychiatric hospital, crossseccional study, physical restraint, aggression.

\begin{abstract}
Objective: To evaluate the use of restraints in a large psychiatric hospital in Rio de Janeiro city, Brazil. Methods: A survey was carried on in August 2009. Data on sex, age, diagnostic, use and duration of restraints were collected from patients' notes. Result: Sample consisted on 66 patients, $59 \%$ women, with mean age of 44 years. During the study $24 \%$ of the patients were restrained at least once, but registering details of the procedure was not usual. There were no important adverse events. No associations were observed between restraints and sociodemographic and clinic variables. Conclusions: The use of restraints is a consistent practice in Rio de Janeiro's psychiatric hospitals: the same proportion was observed in three hospitals in distinct periods, 2001 and 2004, and this finding is in an intermediate position in relation to other studies results. There are no randomized trials to properly evaluate the benefits and risks of this practice, but the efficacy and safety of this intervention should be subject to the same scientific scrutiny as other treatments.
\end{abstract}

1 Fundação Oswaldo Cruz (Fiocruz), Instituto Nacional de Controle de Qualidade em Saúde (INCQS). 2 Nottingham University, United Kingdom (UK), Cochrane Schizophrenia Group. 3 Fiocruz, Escola Nacional de Saúde Pública.

Endereço para correspondência: Gisele Huf Av. Brasil, 4365, Manguinhos - 21040-900 - Rio de Janeiro, RJ Telefone: (21) 3865-5112 E-mail: gisele.huf@incqs.fiocruz.br 


\section{INTRODUÇÃO}

Estudos epidemiológicos demonstraram que pessoas com transtornos mentais são propensas a desenvolverem quadros de agressividade e agitação', e 18\% a 25\% delas apresentam esse comportamento enquanto internadas. Entre os pacientes esquizofrênicos, 10\% a 45\% exibem comportamento agressivo ou ameaçador, principalmente em virtude de sintomas psicóticos agudos durante a hospitalização².

A grande maioria das pessoas com transtornos mentais, entretanto, não é violenta. A violência raramente aparece de modo súbito 1,3, e sinais de alerta são frequentes. No entanto, esses sinais podem ser difíceis de identificar e interpretar ${ }^{3}$. Frequentemente, agressão e violência se seguem a um período de aumento de tensão. Num cenário típico, primeiro a pessoa se mostra irritada, então resiste a ordens e, finalmente, confronta os demais e se torna declaradamente violenta'.

Quando pacientes psicóticos ficam agitados ou agressivos, há necessidade urgente de um tratamento que estabilize o seu estado mental e reduza o risco de machucarem a si próprios ou alguém da equipe hospitalar ${ }^{4,5}$. Contenção física e isolamento em ambiente apropriado, como um quarto vazio, são intervenções usadas para lidar e controlar esses comportamentos violentos na psiquiatria ${ }^{3,6}$. A contenção envolve técnicas desenhadas para confinar os movimentos do corpo do paciente, ou seja, restringir a habilidade do paciente de se mover usando dispositivos diferentes ${ }^{3,7}$, como algemas ou amarras de algodão ou couro ${ }^{8}$. O uso de contenção, para alguns autores, geralmente tem um efeito desejado imediato: a situação ameaçadora é resolvida e não se repete com a liberação do paciente ${ }^{9}$. Portanto, a contenção física seria a resposta final ao comportamento violento iminente, quando medidas menos restritivas falharam ou não são apropriadas' 1 .

O manual da American Psychiatric Association, baseado em um consenso de especialistas, sugere as seguintes intervenções, nesta ordem: (i) tentar acalmar o paciente verbalmente; (ii) fazer um diagnóstico obtendo o histórico, medindo sinais vitais, exame físico e laboratorial, incluindo uma análise de urina. Quando a tentativa de acalmar verbalmente uma pessoa agressiva falha, existe um número limitado de maneiras para controlar a situação e garantir que todos estejam a salvo: (i) tratar o paciente com benzodiazepínico via oral, a não ser que o paciente esteja visivelmente psicótico; (ii) usar um antipsicótico via oral ou IM e (iii) amarrar ou isolar o paciente ${ }^{10}$. Segundo esse manual, maiores riscos de dano ao paciente estão associados às seguintes intervenções, em ordem crescente: (i) medicação voluntária; (ii) medicação de emergência; (iii) combinação de medicação mais contenção física, isolamento; (iv) observação sem intervenção; (v) contenção física e (vi) nenhuma intervenção ${ }^{10}$. No entanto, esse manual também afirma que a contenção, quando usada apropriadamente, pode ser uma medida capaz de proteger a vida, evitar lesões e diminuir a agitação do paciente, opinião essa compartilhada por outros autores ${ }^{1,3,11}$.

O desafio nos quadros de agitação e agressividade é aplicar uma abordagem que garanta a segurança, mantendo a dignidade individual e evitando o uso inapropriado da contenção, pois também se reconhece que o uso dessas técnicas pode gerar efeitos físicos e psicológicos danosos ao paciente ${ }^{13,9,11,12}$. Em 1994, uma comissão constituída pelo U.S. Department of Health and Human Services ${ }^{13}$ relatou 111 mortes de pacientes em contenção física no período de 10 anos, terminando em 1993. No Reino Unido, um manual elaborado pelo National Institute for Health and Clinical Excellence ${ }^{14}$ afirma que a prática mais frequente é o uso de isolamento e contenção manual dos pacientes e recomenda que a contenção física seja utilizada como último recurso. No entanto, reconhece que inexistem estudos adequadamente conduzidos nos quais se possam amparar essas recomendações.

O uso da contenção física é comum em vários países, mas, apesar do que recomendam os manuais, sua frequência em serviços psiquiátricos de emergência varia consideravelmente. A tabela 1 apresenta um resumo da prevalência de uso de contenção física em diversos países 2,9,12,15-20.

Tabela 1. Prevalência de uso de contenção física em diversos países

\begin{tabular}{|c|c|c|}
\hline País & Prevalência (\%) & Artigo citado \\
\hline Estados Unidos & 16 & Bell e Palmer, $1983^{15}$ \\
\hline Estados Unidos & 59 & Beck et al., $1991^{16}$ \\
\hline Estados Unidos & 33 & Lavoie, $1992^{17}$ \\
\hline Estados Unidos & 8,5 & Allen e Currier, $2004^{18}$ \\
\hline Estados Unidos & $7,1-9,9$ & Craw e Compton $2006^{19}$ \\
\hline Itália & 0,25 & Raja e Azzoni, $2005^{2}$ \\
\hline Índia & 15 & Alexander et al., $2004^{20}$ \\
\hline Finlândia & 3,8 & Kaltiala-Heino et al., $2000^{9}$ \\
\hline Alemanha & 49,9 & Martin et al., $2007^{12}$ \\
\hline Suíça & 20,7 & Martin et al., 2007 ${ }^{12}$ \\
\hline
\end{tabular}

Em hospitais psiquiátricos do Rio de Janeiro, o uso de contenção física em pessoas agitadas ou violentas é uma prática comum e aceita, enquanto o isolamento é muito menos comum. Amarras fortes e macias de algodão são prescritas para tornar a situação segura antes do início da ação da medicação. A contenção pode ser retirada após a medicação produzir efeito ou quando se avalia que não existe mais perigo para as pessoas envolvidas. Os estudos TREC (Tranquilização Rápida - Ensaio Clínico) conduzidos no Rio de Janeiro em três hospitais psiquiátricos ${ }^{21,22}$ estimaram que $24 \%$ dos pacientes agressivos foram contidos em algum momento durante as duas primeiras horas após a admissão, e não houve relato de dano para o paciente decorrente desse procedimento. 
Alguns estudos sugerem que os pacientes e a equipe preferem o uso de medicação à prática de contenção física e isolamento. Aparentemente, as medicações são percebidas como um tratamento, enquanto a contenção e o isolamento são percebidos como punição $7,11,23$. De qualquer forma, há um consenso de que o processo de aplicação de contenção física deva ser realizado de maneira segura e eficaz, de modo que funções vitais, como respirar, não sejam afetadas.

Uma revisão sistemática foi conduzida pela Cochrane Collaboration sobre o uso de contenção física e isolamento ${ }^{3}$. Os autores buscaram estudos randomizados cujo foco era avaliar a eficácia e os efeitos adversos do uso de contenção ou isolamento, assim como estratégias designadas para reduzir o uso de contenção ou isolamento no tratamento de doenças mentais graves (excluindo estudos com pacientes com demência ou que faziam abuso de álcool ou drogas, ou ainda sobre contenção de idosos e pessoas confusas). Nenhum estudo foi identificado. Em uma época em que a medicina baseada em evidências ganha cada vez mais espaço, é surpreendente que uma prática a tal ponto disseminada, inclusive recomendada por manuais conceituados, não tenha sido avaliada por meio de investigações metodologicamente apropriadas.

O objetivo deste estudo foi investigar o uso da contenção física em um hospital psiquiátrico de grande porte. Pretende-se, ainda, que esses dados forneçam subsídios para o desenho de um ensaio clínico pragmático sobre a efetividade e riscos dessa prática.

\section{MÉTODOS}

\section{Desenho, local e participantes}

Um inquérito foi conduzido em um hospital de grande porte do Rio de Janeiro. Esse hospital cobre uma área programática de aproximadamente 600 mil habitantes, possui cerca de 100 leitos e funciona 24 horas por dia. A média mensal de atendimentos na emergência é de cerca de 1.300 e aproximadamente $11 \%$ desses originam internações. Todos os pacientes internados nas enfermarias (com exceção das enfermarias de alcoolismo) e leitos diagnósticos (enfermarias de curta permanência) no dia 17 de agosto de 2009 foram investigados retrospectivamente desde o dia de sua internação (ou até quatro semanas antes dessa data) e prospectivamente até o dia da alta (ou até quatro semanas após essa data).

\section{Coleta de dados}

As informações foram coletadas mediante consulta ao prontuário médico desses pacientes.

\section{Variáveis de interesse}

Sexo, idade, diagnóstico de entrada, primeira internação, se internado sob coerção. Ocorrência de contenção física, tem- po de duração, número de observações durante o procedimento, ocorrência de eventos adversos.

\section{Análise de dados}

As análises incluíram o cálculo de médias, proporções e medidas de dispersão. Intervalos de confiança de 95\% também foram estimados. As análises foram realizadas utilizando-se o programa estatístico EPI INFO 6.04.

\section{Aspectos éticos}

Este estudo foi aprovado pelo comitê de ética do hospital em questão.

\section{RESULTADOS}

\section{Descrição da amostra}

No dia 17 de agosto de 2009 havia 66 pacientes internados nos leitos diagnósticos e enfermarias selecionadas, assim distribuídos: 7 (10,6\%) pacientes no leito diagnóstico masculino, 14 (21,2\%) no leito diagnóstico feminino, 20 (30,3\%) na enfermaria masculina e $25(37,9 \%)$ na enfermaria feminina. A maior parte dos pacientes era do sexo feminino (59,1\%). A média de idade era de 44,4 anos $(\mathrm{dp}=12,4)$, variando de 17 a 81 anos.

A maioria dos pacientes $(59,1 \%)$ chegou ao hospital trazida por familiares ou pela polícia, e apenas 6 pacientes $(9,1 \%)$ foram internados no período entre 22 e 8 horas. Três pacientes $(4,5 \%)$ estavam sabidamente internados pela primeira vez. A maioria dos pacientes $(59,1 \%)$ foi diagnosticada como esquizofrênica (F20) no momento de sua entrada no hospital.

\section{Uso de contenção física}

As informações sobre o uso de contenção física foram obtidas nos prontuários dos pacientes e livros de ocorrências de cada uma das unidades (LDF, LDM, EF e EM). Muito pouco foi encontrado no registro dos livros, não sendo prática corrente no hospital o registro detalhado do procedimento, sequer de sua duração. Se algum paciente foi mantido em isolamento durante o período, isso tampouco foi registrado no prontuário ou livros de ocorrência. Por outro lado, também não ocorreram eventos adversos importantes, cujo registro teria sido inevitável, como a necessidade de chamar o plantonista por qualquer razão.

Durante o período estudado, 16 pacientes $(24,2 \%)$ foram contidos no leito pelo menos uma vez; 3 pacientes foram contidos pelo menos duas vezes; 2 pacientes, pelo menos quatro vezes; e 1 indivíduo foi contido pelo menos cinco vezes. A tabela 2 apresenta a relação entre o uso de contenção e algumas variáveis sociodemográficas e clínicas. Nenhuma dessas associações foi estatisticamente significativa. 
Tabela 2. Uso de contenção física segundo variáveis sociodemográficas e clínicas

\begin{tabular}{ll}
\hline Variáveis & Pacientes contidos pelo menos uma vez \\
\cline { 2 - 2 } & $\mathrm{N}$ \\
\hline Sexo & \\
Masculino & $9 / 16$ \\
Feminino & $7 / 16$ \\
Faixa etária & \\
18-35 & $4 / 16$ \\
$36-50$ & $10 / 16$ \\
$51+$ & $2 / 16$ \\
Diagnóstico & \\
F20 & $8 / 16$ \\
Outros & $3 / 16$ \\
Ignorado & $5 / 16$ \\
Primeira internação & \\
Sim & $1 / 16$ \\
Não & $10 / 16$ \\
Ignorado & $5 / 16$ \\
Situação na internação & \\
Desacompanhado & $1 / 16$ \\
Familiares & $6 / 16$ \\
Polícia & $3 / 16$ \\
Ignorado & $6 / 16$ \\
\hline
\end{tabular}

Como alguns pacientes foram contidos mais de uma vez, houve um total de 24 contenções no período do estudo. Com exceção de um episódio em que um paciente foi contido por apresentar convulsão, todos os outros episódios de contenção tiveram como causa a agitação do paciente. Quanto ao horário em que essa intervenção foi aplicada, 6 se deram entre 8 e 17 horas; 12, entre 17 e 8 horas; e em 6 episódios o período é ignorado.

Embora não houvesse registro do fato, não é possível afirmar que nenhum paciente tenha sido posto em isolamento, uma vez que existem espaços apropriados nas enfermarias e leitos diagnósticos, e durante a coleta de dados foram observados dois pacientes (que não faziam parte da amostra investigada) nessa situação.

\section{DISCUSSÃO}

A principal causa para que um paciente fosse contido foi agitação psicomotora. Este estudo também estimou que um quarto dos pacientes que estavam internados nas enfermarias e leitos diagnósticos do hospital no dia 17 de agosto de 2009 foi contido pelo menos uma vez durante sua internação. Esse mesmo percentual foi observado nos estudos TREC ${ }^{21,22}$, conduzidos em três hospitais psiquiátricos do Rio de Janeiro, e situa-se em uma faixa intermediária em relação aos resultados observados em outros países (Tabela 1). Segundo dados de Martin et al.'2, a média de duração do isolamento ou contenção foi cinco vezes mais alta nos hospitais suíços (48,7 e 55 horas para contenção e isolamento, respectivamente) em comparação com os alemães (9,6 e 7,4 horas para contenção e isolamento, respec- tivamente). Para esse autor, a variação no uso da contenção física pode ser uma expressão das tradições locais, e seu uso parece depender mais da equipe do que das características dos pacientes. Infelizmente, não havia informação disponível em nosso estudo sobre a duração da contenção.

Pelo menos metade dos episódios ocorreu após as 17 horas, quando os médicos da rotina já não estavam mais presentes. Embora sem significância estatística, houve um maior percentual de homens contidos, o que também reproduz os achados dos estudos TREC ${ }^{21,22}$. Outras diferenças também não foram estatisticamente significativas ( $p>0,05)$, mas deve-se assinalar que o pequeno número de eventos (16 pacientes contidos) pode ter comprometido o poder estatístico das comparações entre subgrupos de pacientes.

Uma das principais limitações deste estudo é o uso apenas dos dados presentes nos registros feitos em prontuários e livros de ocorrência. Se por um lado esse registro é confiável no que se refere à ocorrência de contenção física, ele é extremamente limitado quanto às circunstâncias em que ela ocorre, como sua duração, a impressão da equipe ou do paciente. Quanto ao isolamento, este parece ser bem menos utilizado do que a contenção no leito, e o seu registro não faz parte da rotina da equipe de enfermagem. Também é alto o percentual de informações incompletas, como o registro da situação de entrada, se primeira internação, entre outras. Apenas um estudo com desenho prospectivo poderia dar conta dessas carências. No entanto, a total ausência de dados provenientes de estudos realizados no Brasil acentua a importância dessa investigação, especialmente por se tratar de um estudo preparatório para uma investigação mais criteriosa da efetividade e segurança no uso da contenção física.

\section{CONCLUSÃO}

A prática de contenção física de pacientes agitados parece consistente nas emergências psiquiátricas da cidade do Rio de Janeiro, mesmo quando estimadas em três momentos distintos: 2001, 2004 e 2009. As equipes de enfermagem são bastante seguras em relação ao procedimento, e não ocorreram eventos adversos importantes durantes as observações. Entretanto, nota-se que o registro das observações é um tanto negligenciado e muito pouco se pode encontrar sobre o período de contenção no prontuário dos pacientes ou no livro de ocorrência das enfermarias e leitos diagnóstico.

Uma revisão sistemática da Cochrane Collaboration ${ }^{3}$ não encontrou estudos randomizados para se proceder a uma avaliação objetiva dos benefícios e riscos dessa prática. A abordagem terapêutica de pessoas tão enfermas a ponto de serem incapazes de controlar sua impulsividade e violência não deve mais ser negligenciada e a segurança e eficácia dessa intervenção deve ser objeto do mesmo escrutínio científico normalmente destinado aos outros tratamentos. 


\section{AGRADECIMENTOS}

\section{Agradecemos a Antônio Vitor Barbosa pelo acesso aos dados de atendimento do hospital onde a pesquisa foi realizada.}

\section{REFERÊNCIAS}

1. Wand TC, Coulson K. Zero tolerance: a policy in conflict with current opinion on aggression and violence management in health care. Aust Emerg Nurs J. 2006;9(4):163-70.

2. Raja M, Azzoni A. Hostility and violence of acute psychiatric inpatients. Clin Pract Epidemol Ment Health. 2005;1:11.

3. Sailas E, Fenton M. Seclusion and restraint for people with serious mental illnesses. Cochrane Database Syst Rev. 2000(2):CD001163.

4. Hovens JE, Dries PJ, Melman (T, Wapenaar RJ, Loonen AJ. Oral risperidone with lorazepam versus oral zuclopenthixol with lorazepam in the treatment of acute psychosis in emergency psychiatry: a prospective, comparative, open-label study. J Psychopharmacol. 2005;19(1):51-7.

5. Breier A, Meehan K, Birkett M, David S, Ferchland I, Sutton V, et al. A double-blind, placebo-controlled dose-response comparison of intramuscular olanzapine and haloperidol in the treatment of acute agitation in schizophrenia. Arch Gen Psychiatry. 2002;59(5):441-8.

6. Steinert T, Eisele F, Goeser U, Tschoeke S, Uhlmann C, Schmid P. Successful interventions on an organizational level to reduce violence and coercive interventions in in-patients with adjustment disorders and personality disorders. Clin Pract Epidemol Ment Health. 2008:4:27.

7. Hodge AN, Marshall AP. Violence and aggression in the emergency department: a critical care perspective. Aust Crit Care. 2007;20(2):61-7.

8. Migon MN, Coutinho ES, Huf G, Adams CE, Cunha GM, Allen MH. Factors associated with the use of physical restraints for agitated patients in psychiatric emergency rooms. Gen Hosp Psychiatry. 2008;30(3):263-8.

9. Kaltiala-Heino R, Korkeila J, Tuohimaki C, Tuori T, Lehtinen V. Coercion and restrictions in psychiatric inpatient treatment. Eur Psychiatry. 2000;15(3):213-9.

10. Allen MH, Currier GW, Hughes DH, Reyes-Harde M, Docherty JP. The Expert Consensus Guideline Series. Treatment of behavioral emergencies. Postgrad Med. 2001(Spec No):1-88; quiz 89-90.
11. Wynn R. Medicate, restrain or seclude? Strategies for dealing with violent and threatening behaviour in a Norwegian university psychiatric hospital. Scand J Caring Sci. 2002:16(3):287-91.

12. Martin V, Bernhardsgrutter R, Goebel R, Steinert T. The use of mechanical restraint and seclusion in patients with schizophrenia: a comparison of the practice in Germany and Switzerland. Clin Pract Epidemol Ment Health. 2007;3:1.

13. NASMHPD. Reducing the use of seclusion and restraint. In: U.S. Department of Health and Human Services; 1999.

14. NICE. Violence: the short-term management of disturbed/violent behaviour in psychiatric in-patient settings and emergency departments. In: National Collaborating Center for Nursing and Supportive Care. Clinical Guideline 25. London; 2005.

15. Bell CC, Palmer J. Survey of the demographic characteristics of patients requiring restraints in a psychiatric emergency service. J Natl Med Assoc. 1983;75(10):981-7.

16. Beck JC, White KA, Gage B. Emergency psychiatric assessment of violence. Am J Psychiatry. 1991;148(11):1562-5.

17. Lavoie FW. Consent, involuntary treatment, and the use of force in an urban emergency department. Ann Emerg Med. 1992;21(1):25-32.

18. Allen MH, Currier GW. Use of restraints and pharmacotherapy in academic psychiatric emergency services. Gen Hosp Psychiatry. 2004;26(1):42-9.

19. Craw J, Compton MT. Characteristics associated with involuntary versus voluntary legal status at admission and discharge among psychiatric inpatients. Soc Psychiatry Psychiatr Epidemiol. 2006;41(12):981-8.

20. Alexander J, Tharyan P, Adams C, John T, Mol C, Philip J. Rapid tranquillisation of violent or agitated patients in a psychiatric emergency setting. Pragmatic randomized trial of intramuscular lorazepam v. haloperidol plus promethazine. Br J Psychiatry. 2004:185:63-9.

21. Rapid tranquillisation for agitated patients in emergency psychiatric rooms: a randomized trial of midazolam versus haloperidol plus promethazine. BMJ. 2003:327(7417):708-13.

22. Huf G, Coutinho ES, Adams CE. Rapid tranquillization in psychiatric emergency settings in Brazil: pragmatic randomized controlled trial of intramuscular haloperidol versus intramuscular haloperidol plus promethazine. BMJ. 2007;335(7625):869.

23. Gilburt H, Rose D, Slade M. The importance of relationships in mental health care: a qualitative study of service users' experiences of psychiatric hospital admission in the UK. BMC Health Serv Res. 2008;8:92. 